others were sacrificed after ligation of the common carotid arteries on each side.

Some of these animals with ligation of the commonc arotid arteries were administered with predonine solution intravenously in varying doses and were sacrificed after several hours or days for histologic examination of brain tissues and meninges.

\title{
Results
}

The result of these histological examination was summarized as follows:

1. In the group of rabbits with bilateral carotid ligations, the edematous finding of brain substance was significant until 24 hours after ligation and then decreased gradually. But it was also characteristic that edema was found as yet after eight days.

2. In the other group of rabbits with bilateral carotid ligation and administration of Predonine intravenously, the edematous finding of brain substance was noted as in the first group. But the findings after 18 hours were apparently distinguished from the former in diminution of edema.

3. Concerning the dosage it was concluded that the optimum dose was found between 1 and $5 \mathrm{mg}$ per $\mathrm{kg}$. experimentally.

\section{Pituitary-Adrenocortical Response after the Cranitomy of Brain Tumor, and its Suppression by Glucocorticoid}

\author{
Koji Nakamura and Kinjiro Iwata \\ First Department of Surgery Nagoya University School of Medicine
}

The suppressive effects of the glucocorticoid therapy after the craniotomy upon the pitnitary-adrenocortical function were discussed. We have applied dexamethasone to prevent cerebral edema after the craniotomy especially of the brain tumor cases. This study was made in the series of brain tumor operation of our clinic from 1962 to the beginning of 1964 .

This steroid treatment was done under constant schedule that $16 \mathrm{mg}-$ $24 \mathrm{mg}$ of dexamethasone was administered on the operation day and $16 \mathrm{mg}$ on the 1 st post-operative day, $12 \mathrm{mg}$ on the 2 nd day, and then decreased gradually down to $1 \mathrm{mg}$ on the 6 th day.

Without steroid treatment, urinary total $17 \mathrm{OHCS}$ of the brain tumor cases increased higher than that of non-tumorous intracranial diseases.

When dexamethasone was given following our schedule of steroid treatment, urinary total $17 \mathrm{OHCS}$ of the craniotomy of the tumor cases decreased 
to the level of the craniotomy of non-tumorous intracranial diseases.

These data show the interesting facts that adrenocortical over-response on the craniotomy of brain tumor cases are suppressed successfully.

Even when unusually high dosage of dexamethasone was given during the postoperative 7 days, lasting more than 4 weeks after the craniotomy, no apparent adrenocortical insufficiency was observed; normal reaction to the adrenocortical reserve test (response to ACTH-Z 40 I.U. for 3 days) and suppression test (response to the dexamethasone $2 \mathrm{mg} / \mathrm{day}$ for three days) were observed as well.

\title{
38. Changes of Renal Function, Water and Electrolytes Following Cerebral Injury
}

\author{
S. Endo, K. Endo, T. Yamada, F. Furukawa, \\ I. Ogawa and Y. AKiba \\ 2nd Department of Surgery, Fukushima Medical College \\ (Director: Prof. S. Endo)
}

Correlation between both renal and endocrine functions, and cerebral injury divided into five groups, i.e. 1, 2, 3, 4 degrees and other sequelae is clarified through the measurement of G.F.R., R.P.F., F.F., N.P.N., Electrolytes in blood and urine, G.P.T. and G.O.T. in blood serum, and 17-O.H.C.S. and $17-$ K.S. in urine.

The results obtained are as follows;

1) getting lower of renal functions in parallel with severity of injury.

2) almost normal value of electrolytes in blood.

3) decrease of $\mathrm{NaCl}$ and increase of $\mathrm{K}$ discharged into urine early after injury.

4) rising of 17-K.S. and 17-O.H.C.S. in urine, and G.O.T. and G.P.T. in serum also early after injury.

Thus it is revealed that renal dysfunction runs parallel to the severity of cerebral injury associated with some affection of central nervous and endocrine system. 